\title{
Feminización de la pobreza y perspectiva de género
}

\author{
José María Tortosa \\ Universidad de Alicante \\ jm.tortosa@ua.es
}

\section{Resumen}

El concepto «feminización de la pobreza» se refiere al hecho de que la pobreza afecta, con más frecuencia, a las mujeres. Sin embargo, esta "feminización» no se encuentra reflejada en las mediciones convencionales, e incluso cuando se producen cuantificaciones de la misma no se introduce un elemento fundamental para explicar dicha «feminización», a saber, la posición de la mujer en la estructura social y su vulnerabilidad estructural. Es decir, la perspectiva de género.

Palabras clave: pobreza, medición de la pobreza, feminización de la pobreza, perspectiva de género.

\begin{abstract}
The concept of the "feminization of poverty» means that poverty largely affects women. Nevertheless, this "feminization" is not reflected in conventional measurements of poverty and, even when it is quantified, one of the fundamental elements for explaining this "feminization» is not taken into account: namely, the position of women in the social structure and their structural vulnerability. That is to say, the gender perspective.
\end{abstract}

Keywords: poverty, poverty measurement, feminization of poverty, gender perspective. 


\section{Introducción}

Una primera aproximación a la cuestión de la «feminización de la pobreza» se obtiene de la segunda edición de un diccionario internacional sobre la pobreza, publicada en 2007 (VV.AA., 2007). En ella se indica que «la tesis [de la feminización de la pobreza] sostiene que, como efectos de la recesión y los recortes en el gasto público, las mujeres están cada vez más representadas entre los pobres del mundo. [... ] Se ven afectados, en particular, los hogares monoparentales y los compuestos por una sola persona anciana.» $\mathrm{Al}$ margen de su contenido eurocéntrico — es decir, contemplar por mundial lo que es local (anglosajón) —, la definición contiene dos elementos que deben retenerse: por un lado, el carácter cuantitativo de dicha «feminización», de tal modo que, tomada literalmente, significaría únicamente un aumento de la cantidad de mujeres entre los pobres «del mundo». Por otro, la concreción, igualmente eurocéntrica, en determinados tipos de hogares, más afectables por los cambios económicos y las políticas sociales, es decir, los hogares monoparentales con cabeza de familia femenino y los formados por ancianas solas. Se trata de hogares (porque casi todo el tiempo se habla de hogares, no de personas) a los que los recortes en el gasto público pueden privar de la «red de seguridad» de unos servicios sociales cuya función es, precisamente, la de evitar la caída en la pobreza de los más débiles.

Esta tesis fue ampliamente seguida en las décadas de 1970 y 1980 y obtuvo, prosigue el diccionario, críticas casi inmediatas. La primera y más inaplazable fue la de negar la novedad del fenómeno: según los autores allí citados, la proporción de mujeres entre los pobres habría permanecido inalterada durante mucho tiempo y no supondría, por tanto, novedad alguna. La segunda, más sugestiva, fue la de distinguir entre la posible feminización de la pobreza en términos cuantitativos y empíricos, por un lado, y, por otro, la percepción del fenómeno que sí podría ser nueva, fruto, entre otros factores, del trabajo realizado por el movimiento feminista apuntando a un hecho que ya existía, pero del que no se había levantado acta desde el androcentrismo de las ciencias en general, y de las ciencias sociales en particular (Chant, 2003: cap. 1). La tercera y más radical consistía en mostrar que, aunque se produjesen nuevas cuantificaciones, éstas no conseguían sacar a la palestra un hecho mucho más importante y que debía explicarse: que la pobreza de las mujeres dentro de su hogar seguía siendo algo escondido, no visible, pero no por ello inexistente.

Sí parece, en cualquier caso, que la definición apuntaba a un factor capitalः el efecto desigual que las crisis económicas acarrean sobre la composición sexual de la pobreza. Precisamente por ello, el asunto cobra particular relevancia en la actual crisis, iniciada en 2007 y reconocida en 2008 a escala mundial. Lo cierto es que, la Comisión de la Situación Jurídica y Social de la Mujer, organismo que forma parte del sistema de las 
Naciones Unidas, reconocía (2009) que «las crisis financieras y económicas» tenían «efectos particulares sobre las cuestiones de género y constituían una carga desproporcionada para las mujeres, en particular las mujeres pobres, migrantes y pertenecientes a minorías». El diagnóstico era el siguiente:

Aun cuando tanto las mujeres como los hombres se veían afectados por las pérdidas de empleos, las mujeres solían ser despedidas primero, dado que los hombres eran considerados tradicionalmente los principales sostenes de la familia. En tanto que en algunos países los sectores dominados tradicionalmente por los hombres estaban experimentando graves pérdidas en el terreno laboral, en otros países las pérdidas de empleos se registraban principalmente en los sectores ocupados mayoritariamente por las mujeres. Los recortes del gasto público en el sector social incidían negativamente en la «economía asistencial», agravando las responsabilidades hogareñas y asistenciales de las mujeres. Los participantes observaron que las mujeres del sector informal se veían particularmente afectadas dado que las crisis económicas tendían a reducir en forma significativa la demanda de productos de ese sector.

En general, la Comisión reconocía la «necesidad de integrar una perspectiva de género en los marcos macroeconómicos y a tal fin recomendaron que se realizara un análisis de las políticas económicas y auditorías desde la perspectiva de género. Se recomendó la utilización sistemática de una presupuestación que tenga en cuenta las cuestiones de género como estrategia para responder a las perspectivas de género de la crisis actual.» $\mathrm{Al}$ mismo tiempo, mostraba su preocupación por «el hecho de que si no se contenía la crisis, algunos países no podrían alcanzar los Objetivos de Desarrollo del Milenio, en particular los relativos a la reducción de la mortalidad infantil, la mortalidad materna y la promoción de la igualdad entre los géneros y el empoderamiento de las mujeres».

En consecuencia, existe un problema llamado «feminización de la pobreza», probablemente agravado por la «crisis financiera y económica», que exige ser estudiado y valorado. Si ya antes de esta crisis reciente un 54\% de los africanos encuestados por Gallup (2008) reconocía que no tuvo dinero suficiente para comprar alimentos para su familia (29\% en América Latina, 25\% en Asia - sin China - y 10\% en Europa) y, en una encuesta posterior (Gallup, 2009), un 56\% de los africanos encuestados afirmaba haber padecido momentos de hambre en la familia a lo largo de los últimos doce meses (16\% en América, 9\% en Asia y 3\% en Europa), no es descabellado pensar, a pesar de lo problemáticos que son los datos recién presentados, que algunos porcentajes se hayan incrementado realmente, y que dicho cambio haya afectado de forma especial a las mujeres, aumentando su porcentaje entre los pobres del mundo.

A pesar de lo dicho, las medidas convencionales de medición de la pobreza no sólo no facilitan esa cuantificación, sino que incluso la dificultan, como se va a demostrar a 
continuación (epígrafe 1), para pasar, en los epígrafes 2 y 3 , respectivamente, a presentar las diferencias entre la «feminización de la pobreza» (enfoque cuantitativo tanto en su vertiente sincrónica como diacrónica) y la perspectiva de género (más estructural y más difícil de cuantificar).

\section{Las medidas}

Tres son los grandes grupos de mediciones de la pobreza de los que se puede disponer para efectuar comparaciones a escala internacional. Dos de ellas reflejan las definiciones de pobreza que en su momento diera Amartya Sen: una sería «no tener suficiente» (pobreza absoluta) y la otra «tener menos» (pobreza relativa). A estas dos se añade el intento del Programa de Naciones Unidas para el Desarrollo (PNUD) de ofrecer, mediante el Índice de Pobreza Humana, un acercamiento algo más complejo que permita, por lo menos a primera vista, establecer las mejoras o deterioros de los distintos países en cuanto a la pobreza, tal y como es definida por el PNUD.

\subsection{La pobreza absoluta}

Uno de los Objetivos de Desarrollo del Milenio consistía en reducir el porcentaje de personas que vivieran en la pobreza. Lo que de manera altisonante se anunciaba como «erradicar la pobreza y el hambre» se trasformaba, pragmática y operativamente, en «reducir a la mitad, entre 1990 y 2015, la proporción de personas con ingresos inferiores a 1 dólar por día». Además, para hacer todavía más fácil su logro, se había optado por uno de los medios más citados para cuantificar la pobreza absoluta en el mundo: la de personas que viven con menos de un dólar al día (a paridad de poder adquisitivo), cuando, como se va a comprobar, existían otras medidas alternativas que provenían de la misma fuente. No es ahora momento de incidir en que dicho objetivo, entre los posibles, era el más fácil de conseguir, pues no se planteaba la erradicación sino sólo la reducción del porcentaje de pobres, y no su cifra absoluta; en este sentido, se puede reducir el porcentaje de pobres sin cambiar su número absoluto: basta con incrementar el número de ricos o de miembros de clases medias, dejando inalterado el número de pobres.

Tampoco se trata ahora de reconocer que ni siquiera tan modesto objetivo se vaya a conseguir. Antes de la crisis actual, ya se sabía que no se iba a producir (Naciones Unidas, 2005). Con respecto a los «brotes verdes» anunciados para su consecución poco antes de estallar la crisis, con datos que finalizaban en 2004 y que afectaban, sobre 
todo, a los más pobres de los pobres (Naciones Unidas, 2007), hay motivos más que suficientes para ponerlos en duda, también, probablemente, por parte de sus mismos autores, superados por los acontecimientos posteriores a su informe.

En general, esta pobreza absoluta queda definida como el número de personas que no alcanzan un determinado nivel de ingresos/gastos que se considera mínimo para llevar una vida digna. Y el dólar por persona y día, ya citado, ha sido el indicador más utilizado durante años por el Banco Mundial, hasta que se comenzó a revisar por el mismo Banco (Ravallion, Chen y Sangraula, 2008), produciendo, así, nuevas cuantificaciones sobre esta pobreza absoluta mediante las diferentes «líneas de pobreza» que separarían a los pobres de los que no lo son. De esta forma, el número de pobres oscilaría entre 879 millones, si la línea se establece en 1 dólar por persona y día; 1.399 millones si, en cambio, se hace según el nuevo cálculo de 1,23 dólares, y los 3.140 millones si la línea se sitúa en 2,50 dólares por persona y día (Chen y Ravallion, 2008). El asunto se puede complicar todavía más si se incluye a China entre los países que cuantificar (no es la pobreza «en el mundo», sino en los «países en desarrollo», y China es un país emergente), con lo que, al final, dispondríamos, para 2005, de diez cuantificaciones diferentes para dichos países: usando como «línea de pobreza» 1 dólar, 1,25 \$, 1,45 \$, $2 \$$ y $2,20 \$$, con o sin China. No queda claro en qué queda reducida la pobreza cuando se dispone de diez cifras y sus correspondientes diez porcentajes para medirla.

Yendo a los países ricos, Estados Unidos utiliza un sistema parecido, pero mucho más sofisticado, aunque no sea más que porque, a diferencia del Banco Mundial, se basa en un censo. Periódicamente, la Oficina del Censo de Estados Unidos (U.S. Census Bureau, 2009) publica la «línea de pobreza» para una familia según el número de componentes que tenga (de 1 a 9 o más). En concreto, el Gobierno estadounidense proporciona tres conjuntos de «líneas de pobreza»: una para los «estados contiguos y el distrito de Columbia», otra para Hawai y otra para Alaska, con lo que refleja las diferencias, aunque no sean más que climatológicas, en cuanto al dinero necesario para obtener la «canasta de bienes y servicios» que se consideran mínimos en cada caso. Aplicando estas «líneas», Estados Unidos habrían contado en 2007, oficialmente, con algo más de 37 millones de pobres; es decir, un 12,5 \% de la población.

Sin embargo, y a pesar de su sofisticación, estas líneas de pobreza no son aceptadas universalmente y, de hecho, la misma Oficina del Censo proporciona las cuantificaciones que se obtendrían si se aplicasen otros criterios producidos en el país por universidades, think tanks o instituciones. Curiosamente, todas serían superiores a la oficial (Dalaker, 2005), con lo que hace difícil saber cuántos pobres hay realmente teniendo en cuenta esta medida de pobreza absoluta. No deja de ser sospechoso, en efecto, que las cifras de pobres oficiales sean todas inferiores a las que proporcionan otras instituciones, con las mismas fuentes pero calculadas de manera alternativa. 


\subsection{La pobreza humana}

En el extremo opuesto de las cuantificaciones disponibles, y ampliamente citadas, se encuentra el Índice de Pobreza Humana (IPH), a su vez dividido en dos: el IPH-1, para países en desarrollo, y el IPH-2, para países miembros de la OCDE seleccionados, es decir, excluyendo a determinados países que no encajan con la idea convencional de "país rico». Cada índice está compuesto por dimensiones e indicadores y se calcula por separado, de forma que no hay modo de comparar los resultados de los países ricos con los de los países pobres. El IPH-1 contiene las dimensiones de «una vida larga y saludable», «conocimiento» $y$ «un nivel de vida digno». Por su parte, el IPH-2 incluye «una vida larga y saludable» (aunque el indicador es la probabilidad al nacer de no sobrevivir a los 60 años, mientras que en el IPH-1 es de los 40), «conocimiento» (en el IPH-1 era analfabetismo de adultos, aquí es analfabetismo funcional), «un nivel de vida digno» (que se mide a través del porcentaje de personas por debajo de la línea de pobreza) y la «exclusión social», que se reduce al desempleo de larga duración.

En la última edición consultada del Informe sobre el Desarrollo Humano (PNUD, 2007), que es donde se publican los IDH, a saber, la de 2007-2008, los países ricos mejor situados respecto al IDH-2 eran Suecia, Noruega, Países Bajos, Finlandia, Dinamarca, Alemania, Suiza, Canadá, Luxemburgo y Austria. España ocupaba el puesto 15 y Estados Unidos, el 17. Los últimos lugares, para el IDH-1, estaban ocupados por el Chad (en la peor posición mundial), Malí, Burkina Faso, Etiopía, Níger, Guinea, Sierra Leona, Mozambique, Benín y Guinea-Bissau.

Estos dos índices, así obtenidos, permiten conocer si la situación de un país ha mejorado o empeorado de un año a otro con respecto a los demás países analizados. En efecto, tomando el anterior Informe de 2006, o el próximo, el de 2009, que se presentó en octubre de dicho año (dedicado a las migraciones), es posible saber si un país ha ascendido en la lista de su propio índice, aunque no se puede saber si ha ascendido por mejora de sus indicadores o lo ha hecho por empeoramiento de los que iban por delante en el Informe de referencia. Al contrario, lo mismo puede decirse si un país ha descendido, cosa que sus medios de comunicación, y más si están en oposición al respectivo gobierno, se dedicarán a subrayar. Sin embargo, el descenso puede haber ido acompañado por una mejora general en todas las dimensiones del índice en el país, pero con la particularidad de que otros países podrían haber mejorado todavía más. Es, pues, un ranking y sólo eso. 


\subsection{La pobreza relativa}

Si la pobreza se va a medir en términos monetarios, casi podría decirse que toda pobreza es relativa. Es decir: el dinero necesario para satisfacer las necesidades básicas dependerá de la sociedad en concreto — ¿hay trueque?, ¿autoabastecimiento?, ¿ayuda mutua como factor de evolución, o bien darvinismo?-, y también serán relativas las necesidades a considerar. Así, por ejemplo, la calefacción será fundamental en Alaska e innecesaria en Hawai. A pesar de ello, cuando de lo que se habla no es de «no tener suficiente» sino de «tener menos que otros», es cuando se usa con más motivo la palabra «pobreza relativa».

El Instituto Nacional de Estadística español, para su Encuesta de Condiciones de Vida (INE, 2008), establece que «el umbral de pobreza depende de la distribución de los ingresos por unidad de consumo de las personas (de ahí el término de pobreza relativa, que tiene en cuenta la situación de la población a la que se pertenece). Se fija este umbral en el $60 \%$ de la media de los ingresos por unidad de consumo de las personas».

En otras palabras, la pobreza relativa se mide calculando la media/mediana del consumo/ingresos familiares y estableciendo como «umbral de pobreza» el 40, 50 o $60 \%$ de aquélla (incluido, por tanto, hasta el $25 \%$ ). El INE se inclina por el $60 \%$ de la media de los ingresos, pero otras fuentes proponen opciones diferentes basadas en las otras combinaciones posibles (mitad de la media de ingresos, $40 \%$ de la media de consumo, etc.).

Un informe sobre la inclusión social en España (Observatorio de la Inclusión Social, 2008) recogía algunas de estas estimaciones para la Unión Europea (UE), utilizando el $60 \%$ y el $40 \%$ de la media, con lo que, por ejemplo para España, se podía afirmar que había un 20\% de pobreza moderada y un 8\% de pobreza alta en 2006. Los datos de 2007 no habían producido grandes cambios (Comisión Europea, 2009). También era posible, para el caso español y sus comunidades autónomas, conocer la media y la mediana de los ingresos equivalentes y los umbrales de pobreza de cada una de ellas según se utilizase el $60 \%$ o el $40 \%$ de la mediana, o bien el $25 \%$ de la media (para Cataluña, respectivamente, 8.276, 5.518 y 3.712 euros), lo que permitiría conocer, mediante encuesta de presupuestos familiares, el número de hogares y, por tanto, de personas por debajo de los diferentes umbrales.

Las diferencias en los umbrales de pobreza de una comunidad a otra no parece que hayan creado grandes problemas a las distintas estimaciones basadas en la renta monetaria española. Sin embargo, la heterogeneidad en las desigualdades (porque la «pobreza relativa» es, en última instancia, una medida dependiente de la desigualdad o incluso una medida de la desigualdad de rentas) entre los miembros de la UE, sobre 
todo con las más recientes incorporaciones, ha hecho que se intente revisar a fondo este sistema de medición (Dennis y Guio, 2004), cosa que es probable que se lleve a cabo próximamente.

\subsection{Elementos en común}

No es fácil optar por una u otra de las definiciones anteriores, con sus respectivas mediciones. En realidad, ninguna de ellas responde adecuadamente a la definición de pobreza (insatisfacción involuntaria, permanente y severa de las necesidades bumanas básicas) y la elección de indicadores suele deberse más a facilidades ulteriores para la medición y posterior tratamiento estadístico que a la coherencia de dichos indicadores con la definición.

Por otro lado, todas ellas permiten un rango muy elevado en las estimaciones posibles, con lo que se puede optar por diferentes indicadores en función de la cantidad que se desee encontrar. Tanto en el caso de la pobreza absoluta como en el de la relativa, se pueden hacer cálculos con líneas de pobreza muy heterogéneas y, en el caso de la «pobreza humana» del PNUD, se podrían obtener estimaciones diferentes cambiando, por ejemplo, los umbrales de la esperanza de vida, o el peso del indicador en el índice final. Todo ello al margen de que no permite la comparación entre países pobres y países ricos, cosa que, en la práctica, tampoco puede hacerse con los datos del Banco Mundial (sólo obtenidos para países «en desarrollo») o con algunos de los disponibles sobre pobreza relativa (por definición, Eurostat, sólo para países de la UE.)

De todas maneras, tal y como se han presentado, las diferentes cuantificaciones posibles hacen muy difícil abordar el problema de la «feminización de la pobreza», que no parece estar en sus pretensiones, como puede verse en alguna publicación de Eurostat en la que, a pesar de las referencias a la desigualdad entre los sexos en campos diversos como el educativo y el laboral, no hay ninguna referencia a las mujeres en el capítulo 4 dedicado a «renta, pobreza, exclusión social y [...] consumo» (Eurostat: 2008). Además, dichas cuantificaciones vendrían particularmente dificultadas por la misma andadura metodológica aplicada. Efectivamente, una cosa son los datos agregados (presupuestos familiares, PIB, Índice de Pobreza Humana) que permiten cuantificar cuántos pobres hay o, en su defecto, qué lugar ocupa el país en un ranking de pobreza, y otra cosa son los datos individuales que permitirían saber las diferencias entre personas, es decir, entre los sexos en general, y dentro de la familia en particular. Si, como suele hacerse (Pérez Yruela et alii, 2009), la muestra se realiza a partir de hogares y, de éstos, se eligen los que tienen menos del 60\% de la renta mediana del ámbito del estudio, difícilmente se podrá hablar de «feminización de la pobreza» a no 
ser que, simplemente, se cuenten cuántas mujeres hay en dichas familias y se calcule su porcentaje sobre el conjunto. Pero eso no será pobreza de las mujeres, sino presencia de las mujeres en los hogares pobres.

\section{La feminización de la pobreza}

El Índice de Desarrollo relacionado con el Género (GDI en inglés) que también propone el PNUD no soluciona el problema, incluso utilizando de manera comparada entre varones y mujeres la esperanza de vida al nacer, el analfabetismo y la escolarización y los ingresos personales. Al final, lo que se obtiene es una lista ordenada de mayor a menor. Es algo, pero no mucho, y menos si se constata que lo que se está reflejando es la distancia entre países centrales y periféricos, reflejada incluso en los criterios «coloniales» de clasificación. Se encontrará, en los primeros puestos, a Islandia, Australia, Noruega, Canadá, Suecia, Países Bajos, Francia, Finlandia, Suiza y Reino Unido (España en el puesto 12, Estados Unidos en el 16), y, en los últimos, a Sierra Leona (la peor situación mundial según este cómputo), Guinea-Bissau, Níger, Burkina Faso, República Centroafricana, el Chad, Malí, Mozambique, Etiopía y la República Democrática del Congo. De nuevo un ranking que no dice mucho sobre la situación concreta, si bien los términos relativos pueden ser interesantes.

De todas formas, algunas de las medidas presentadas en el epígrafe anterior sí que han dado paso a cuantificaciones de la desigualdad entre hombres y mujeres en el terreno presente. Así, por ejemplo, y con datos de 1966 a 2007, el U.S. Census Bureau levantaba acta de la mayor presencia constante de mujeres entre los pobres calculados en Estados Unidos, con un 13,8\% de las mujeres y un 11,1\% de los varones, para 2007. Otros estudios han mostrado que en 2005 la tasa de pobreza de familias con sólo padre fue de un $17,6 \%$, mientras que la de las familias con sólo madre fue de un $36,9 \%$ (Thibos, Lavin-Loucks y Martin, 2007). Probablemente, hay que introducir este último dato para explicar el que proporciona la Oficina del Censo.

Lo mismo se podía decir de España, calculado no como pobreza absoluta sino como pobreza relativa. Según la Encuesta de Condiciones de Vida, 2007 (INE, 2008), en ese año un 20,9\% de las mujeres se encontraba en dicha situación, frente al 18,6\% de los varones.

Generalizando, se puede decir que el empobrecimiento de las mujeres es «una ortodoxia global que ya nadie pone en cuestión» (Davids y Van Driel, 2005: 5). El término «feminización de la pobreza», como aumento de pobreza entre las mujeres (Medeiros y Costa, 2008), se introdujo en el vocabulario sobre el desarrollo a partir los años noventa del pasado siglo, sobre todo con la constatación que se hizo en la IV Conferencia 
de Naciones Unidas sobre las Mujeres de ese $70 \%$ de mujeres entre los pobres del mundo, dato, por cierto, de difícil constatación empírica. Lo cierto es que una de las doce áreas críticas de la Plataforma para la Acción de Pekín (BPFA en inglés) incluía la erradicación del «peso persistente y creciente de la pobreza sobre las mujeres», lo cual no obsta para que se reconozcan los fallos metodológicos y las debilidades conceptuales que comporta (Mateo, 2000; Chant, 2006) y, en todo caso, la necesidad de superarlo desde otras perspectivas (Mateo, 2002). En muchos de los trabajos existentes sobre el tema se puede constatar, como también en los trabajos sobre la pobreza en general, un exceso de énfasis en la renta monetaria, en la familia monoparental con cabeza de familia femenina, y muy escaso en la relación estructural entre varones y mujeres.

A pesar de todo, es ya una «ortodoxia» afirmar que hay más mujeres pobres que varones pobres; el constatar que ha sido así desde que se tienen datos sobre el asunto, y el reconocer que la situación podría empeorar en condiciones de crisis económica. Cuando hablamos de «ortodoxia» no nos referimos a un juicio sobre la verdad o falsedad de estas afirmaciones, sino sobre su difusión en contextos académicos y políticos.

\section{Perspectiva de género}

Carmen de Elejabeitia (1993: 176) indica dónde radica el problema de fondo al decir que «la situación de las mujeres no es la de desigualdad en relación a los hombres, sino la de discriminación. La no discriminación por razón de sexo ha sido el primer objetivo a conquistar por el feminismo hasta los años 70 y hoy concentra la atención y los esfuerzos por parte del que podemos considerar feminismo institucionalizado. La cuestión es que discriminación e igualdad no pueden estar en un mismo discurso, pertenecen a dos planos situacionales distintos y por eso nada dice la discriminación de lo que pueda significar la igualdad, simplemente es su meta, su punto de llegada».

La desigualdad ante la pobreza — que es lo que vendría a ser la «feminización de la pobreza» en su perspectiva estática (en su perspectiva dinámica sería el aumento de dicha desigualdad) - puede ser vista desde el punto de vista del «desfase» entre varones y mujeres. El Índice de Desfase de Género Global (GGGI en inglés), presentado por el informe Global Gender Gap Report (Hausmann, Tyson y Zahidi, 2008) en el Foro Económico de Davos de 2008, está construido a partir de la agregación de conceptos como empoderamiento político, participación económica, logros educativos y salud. El Índice vuelve a situar, con alguna excepción, a parecidos países en los primeros puestos -Noruega, Finlandia, Suecia, Islandia, Nueva Zelanda, Filipinas, Dinamarca, Irlanda, Países Bajos y Letonia (España en el puesto 17, y Estados Unidos en el 27)-, pero cambia notablemente en los últimos puestos si se compara con los ranking ya 
presentados, a saber, el Yemen (en la peor de las situaciones), el Chad, Arabia Saudita, Pakistán, Benín, Marruecos, Egipto, Turquía, Etiopía y Bahrein. En este caso, el sesgo en la construcción del indicador refleja no sólo diferencias políticas y económicas (centro-periferia), sino también culturales. En todo caso, tiene en común con el IDH, el IPH y el GDI el que sólo permite conocer mejoras y deterioros relativos con respecto a los restantes países analizados. Es interesante, de todos modos, que, según dicho informe, se constate que, entre 2007 y 2008, el desfase entre varones y mujeres se está ampliando en 41 países, mientras que se está reduciendo en 87 de los 128 analizados.

Sin embargo, como indica Elejabeitia en el texto ya citado, es preciso, para entender el fenómeno, pasar de la desigualdad (cantidades diferentes de una determinada variable, al fin y al cabo) a la discriminación y la exclusión (Martínez Román, 2001; Mateo, 2001), para lo cual la idea de las violencias puede resultar útil.

Se tiene, para empezar, la violencia directa, física, la violencia de género perpetrada contra las mujeres por ser mujeres (Espinar y Mateo, 2007). No es este el momento de adentrarse en el tema, pero sí de constatar que, en términos generales, la violencia en la familia es perpetrada básicamente por varones, y que las víctimas son básicamente mujeres. Esta violencia es legitimada e incluso propiciada por lo que se puede llamar violencia simbólica o violencia cultural, es decir, el conjunto de ideas, mitos y visiones de la realidad que se pueden encuadrar bajo la etiqueta de machismo o, si se prefiere, androcentrismo.

Ambas violencias tienen su propia lógica y, como se ha dicho, se relacionan entre sí: la violencia cultural legitima o promueve la violencia directa, y la violencia directa busca su legitimación. Y las dos, a su vez, guardan relación con la tercera violencia, la llamada violencia estructural, que, si bien la primera era visible y la segunda puede decirse que era audible, pues suele estar compuesta de palabras, aunque también de imágenes, ésta, la estructural, queda implícita en las relaciones sociales de forma que sólo puede ser inducida a partir de los comportamientos de exclusión y marginación, muchos de ellos cotidianos, en general de periferización, que se observan en las diferentes sociedades.

Esta violencia estructural que afecta a las mujeres (el patriarcado, si se prefiere) no alcanza el mismo grado en todas las sociedades ni lleva, en todas ellas, como ya se ha visto, a los mismos niveles de pobreza o de violencia de género. Desde un punto de vista histórico, esa proporción no tiene por qué haber sido estable, sino que, probablemente, ha cambiado en función de las condiciones del contexto, de tal forma que, en situaciones adversas, es la mujer una de las primeras categorías sociales que sufre los efectos negativos de dichas situaciones. Pero también ha cambiado en proporción a la lucha política entablada por defender a las mujeres vulnerables frente a la periferización a la que se las puede haber sometido y se las somete. Así, por ejemplo, entre 1997 
y 2007 (habrá que ver qué sucede en los años 2008 y 2009) el porcentaje de mujeres en empleos vulnerables, aun siendo alto en particular en el África subsahariana y el Asia meridional, obtuvo visibles reducciones a escala mundial, al igual que las disparidades en niveles de empleo (UNIFEM, 2008).

Tampoco aparece de la misma forma en todos los campos en los que se desarrolla la acción social, tomados en el sentido que le da Pierre Bourdieu al término, que incluiría no sólo la economía y la cultura, sino también la política y, para el presente caso, las instituciones sociales como la familia. De hecho, lo que aquí se ha llamado violencia estructural no tiene por qué presentarse de la misma forma en cada uno de los ámbitos en los que se desarrolla la acción social de los individuos concretos, pudiéndose dar con mayor claridad en unos que en otros. La «sociedad», en efecto, ha de descomponerse en las diferentes esferas de acción que definen los diversos roles asumidos por los actores sociales, que no necesariamente son coincidentes. Puede haber ámbitos, para personas que sufren violencia estructural en otros, en los que no se sufra tal violencia. La insidiosa distinción entre público y privado puede ser útil a este respecto: puede haber violencia estructural en lo «público» (empleo, cargos públicos, reconocimiento) pero no en lo "privado», en este caso familiar (matriarcado difuso, por ejemplo). En algunos países se ha subrayado la existencia de sectores en la sociedad en los que, junto a la relativa ausencia de la mujer en la vida pública, sí es capaz de ejercer un poder muy importante dentro de la familia casi matriarcal, matriarcado doméstico o criptomatriarcado, asuntos no exentos de discusión por su posible carácter androcéntrico, que no es ahora el momento de entrar a analizar.

Un ejemplo, para esta cuestión de los campos, puede obtenerse del Barómetro de diciembre de 2008 del Centro de Investigaciones Sociológicas (CIS, 2008). En él se recogían las respuestas a una pregunta sobre si, en opinión del encuestado o encuestada, era frecuente y cuánto la discriminación o el trato desfavorable por diversos motivos que se enunciaban en la pregunta. El porcentaje más alto de respuestas, en el sentido de ser muy frecuentes, lo obtenía el «origen étnico o racial», seguido de «tener pocos recursos económicos», el «aspecto físico», la «orientación sexual», y sólo en quinto lugar aparecía el «sexo». Raza, clase, sexualidad y sexo serían, por ese orden, los motivos de discriminación observables en España a decir de los encuestados. Sin embargo, si se preguntaba si el encuestado o encuestada había sentido discriminación en el ámbito laboral en los últimos doce meses, daba como respuesta mayoritaria la de no haberlo sentido, pero seguida de inmediato por la discriminación "por su sexo», «por su edad» $y$ «por su origen étnico o racial». Tal vez en el campo de la sociedad en general la discriminación no sea tan perceptible, pero sí es reconocida en el campo económico de las relaciones laborales, y esta discriminación afectará a la probabilidad de obtener un trabajo remunerado, al nivel del salario comparado con el del varón y, seguramen- 
te, también esté afectando a las probabilidades de despido cuando las empresas, para mantener sus beneficios, aprovechen la crisis para hacer recortes en los gastos de personal, que comenzarán por la reducción del empleo femenino.

La vulnerabilidad de las mujeres, es, así pues, el resultado de una posición en la estructura social - contra la que lucha el feminismo- que facilita recibir los efectos negativos del funcionamiento de la sociedad, sea en términos de pobreza (feminización de la pobreza), sea en términos de violencia (violencia de género). A esto se une la idea de discriminación, resultado de una relación estructural entre, por lo menos, dos actores.

Con grados diferentes de vulnerabilidad y de discriminación en las diversas sociedades ( $y$ aun dentro de las mismas), la feminización de la pobreza obtendrá cuantificaciones diferentes, como puede suponerse a partir de la tabla en la que se vuelven a presentar, aunque esta vez de manera comparativa, los diez países mejor situados y los diez peor situados para el Índice de Desarrollo relacionado con el Género (GDI), el Índice de Desfase de Género Global (GGG) y los respectivos Índices de Pobreza Humana (IPH-1 para países seleccionados de la OCDE, o países ricos, e IPH-2 para países «en desarrollo», o países pobres). Se han escrito en cursiva los países que están presentes en los tres ranking (los nórdicos más los Países Bajos, en el caso de los países ricos, y el Chad y Etiopía entre los pobres). Estrictamente, sólo el orden en el GDI y en el GGG puede ser comparado, ya que los datos se han obtenido de la misma forma, cosa que no sucede, como se ha visto, con los dos IPH, lo que no obsta para ver que son países del centro frente a países de la periferia en la parte derecha de la tabla. En todo caso, como también se ha indicado, la posición en estos rangos no significa, mecánicamente, un determinado grado de «feminización de la pobreza», es decir, una determinada cantidad y porcentaje de mujeres entre los pobres del país y, ni mucho menos, un aumento o disminución en el tiempo. De todas formas, es una obviedad añadir que el porcentaje de mujeres pobres en el Chad o en Etiopía va a ser notablemente superior al de los países nórdicos, pero no sólo por su diferente GGG o GDI, sino por muchos otros factores, entre los cuales está el que los primeros se encuentran en el centro y los segundos en la periferia, asunto en el que no conviene detenerse, ya que no explicaría el relativamente bajo puesto que ocupan Estados Unidos. 
Diez primeros y últimos países en las respectivas clasificaciones:

\begin{tabular}{|c|c|c|c|c|c|}
\hline \multicolumn{3}{|c|}{ MEJOR SITUADOS } & \multicolumn{3}{c|}{ PEOR SITUADOS } \\
\hline GDI & GGG & IPH-1 & GDI & GGG & IPH-2 \\
\hline \multirow{2}{*}{ Islandia } & Noruega & Suecia & Sierra Leona & Yemen & Chad \\
Australia & Finlandia & Noruega & Nuinea-Bissau & Chad & Malí \\
Noruega & Suecia & Países Bajos & Níger & Arabia Saudita & Burkina Faso \\
Canadá & Islandia & Finlandia & Burkina Faso & Pakistán & Etiopía \\
Suecia & Nueva Zelanda & Dinamarca & Rentroafricana & Benín & Níger \\
Países Bajos & Filipinas & Alemania & Chad & Marruecos & Guinea \\
Francia & Dinamarca & Suiza & Malí & Egipto & Sierra Leona \\
Finlandia & Irlanda & Canadá & Mozambique & Turquía & Mozambique \\
Suiza & Países Bajos & Luxemburgo & Etiopía & Etiopía & Benín \\
Reino Unido & Letonia & Austria & Depocrática & Bahrein & Guinea-Bissau \\
\hline
\end{tabular}

Puede resultar sugestivo comparar estos rangos con los que Save the Children (2009) proporciona sobre el «estado de las madres» en el mundo a partir de diversos indicadores sobre mortalidad maternal, partos asistidos, anticonceptivos, alfabetismo femenino y presencia femenina en puestos políticos, variables que nada tienen que ver con una «condición maternal» $y$, mucho menos, con una «condición femenina» abstracta, sino que reflejan posiciones en el sistema mundial, diferencias en las políticas públicas, niveles de desigualdad social promovida colectivamente y coyunturas políticas y económicas diferentes de país a país. Los diez países mejor situados, sin sorpresa, serían Suecia, Noruega, Australia, Islandia, Dinamarca, Nueva Zelanda, Finlandia, Irlanda, Alemania y Países Bajos. En el otro extremo de los 158 países analizados estaría Níger, seguido por Sierra Leona, el Chad, Guinea-Bissau, la República Democrática del Congo, Yemen, Sudán, Angola, Eritrea y Yibuti.

De todas formas, para establecer de una manera más concluyente el respectivo grado de «feminización de la pobreza», sería preciso bajar todavía más el nivel de análisis. Por un lado, la cuestión de la «feminización de la pobreza» es difícil de medir si el ámbito de análisis es una entidad política que produce sus estadísticas mediante agregados del tipo PIB (estado, comunidad autónoma, municipio). Tampoco algunos indicadores de cohesión social pueden resultar útiles al respecto, como los que propone el Consejo de Europa, algunos de los cuales pueden resultar útiles para estudiar la situación de las mujeres (Consejo de Europa, 2005: 192-194), pero no para trabajar en el tema de la pobreza. En cambio, por otro lado, puede ser observada de manera algo más cuidadosa si la unidad de análisis es la familia (encuestas de presupuestos familiares, por ejemplo).

Pero, en todo caso, no parece que se vaya a obtener una buena medición de la pobreza desde una perspectiva de género si no se observa la satisfacción de necesidades básicas dentro de la familia, es decir, si la unidad de análisis no es el individuo concreto. 
No es fantasía el suponer que puede haber familias «estadísticamente» no-pobres (es decir, por ejemplo, que tiene un nivel de ingresos superior al $60 \%$ de la renta mediana de su contexto) y, sin embargo, contener miembros con evidente insatisfacción de sus necesidades básicas, es decir, pobres; de igual forma, no es arriesgado suponer que puedan ser la madre o, en general, los miembros femeninos de la misma. Pero para eso no existen buenas «estadísticas», que son las que habría que producir más en la línea de la «deprivación social» que definiera Peter Townsend (Townsend, 1987) junto a su «índice de deprivación» (Townsend, 1979), antes que las basadas en la simplicidad de la monetarización, sea con líneas «absolutas», sea con referencias «relativas». Cierto es que, metodológicamente, son más trabajosas de conseguir, pero pueden responder, si la unidad de análisis es el individuo y no la familia o la circunscripción administrativa, a la cuantificación de la «feminización de la pobreza» que permita fundamentar las reflexiones sobre la perspectiva de género.

\section{Más allá de la perspectiva de género}

Volviendo a los posibles motivos de discriminación utilizados por el CIS, se puede observar que todos los criterios son construcciones sociales que sitúan a la persona clasificada por los mismos en una determinada posición, superior o inferior, en la estructura social. Incluso el «aspecto físico» es resultado cambiante de criterios de «distinción» y clasificación generados en la sociedad. Para lo que aquí se trata, el caso del género (construcción social a partir de la diferencia sexual) es relevante.

Sucede, generalizando, que las diferentes sociedades definen diversos grados de «desfase» entre las personas etiquetadas por criterios importantes para dichas sociedades, y convierten ese «desfase» en un medio de distribuir poder, privilegio y prestigio, de tal forma que, para cada criterio, se obtiene una división de la sociedad que puede simplificarse tal y como George Orwell atribuía en 1984 al personaje de Emmanuel Goldstein: una división, sin duda impresionista pero no por ello menos útil, entre «los de arriba», los «intermedios»y «los de abajo».

Es obvio que estas clasificaciones (sexo, raza, clase, edad) se combinan entre sí de modo que podría construirse un índice de vulnerabilidad individual que situaría en la posición más segura a los varones de raza (pretendidamente) «superior», universitarios, de clase alta y de edad intermedia, y en la más vulnerable a las mujeres, de raza (pretendidamente) «inferior», analfabetas, de clase baja y de edad avanzada. Las variables a utilizar van desde las que ya propuso Wilfredo Pareto en su Trattato di Sociologia Generale a las que usan generalmente en la sociología estadounidense para referirse a la estratificación social. Y también pueden utilizarse los criterios del «capital simbólico» 
descritos por Pierre Bourdieu. Al fin y al cabo, la «feminización de la pobreza» no es de las mujeres en general, sino de las mujeres en específicas situaciones de vulnerabilidad adicional, es decir, situaciones que añaden vulnerabilidad a la que ya supone la discriminación como mujer, haciéndola más visible en unos casos («las de abajo») que en otros («las de arriba») y en unos países (periféricos) que en otros (centrales). De igual modo que la idea de paternidad no es la misma en todas las clases sociales (Valdés, 2009) y en todos los países, la situación de vulnerabilidad no es la misma para todas las mujeres y todos los países y, por tanto, habrá que poner el problema en un contexto algo más general, aunque sin perder la perspectiva de género (o la de clase, o la de «raza»). Ir más allá de la perspectiva de género no significa necesariamente minimizar su importancia para entender las cuantificaciones, por otro lado necesarias si se quiere un mínimo de intersubjetividad, verificabilidad y falsabilidad, que esa es la actividad científica.

\section{Bibliografía}

Chant, Sylvia H. (2003). New contributions to the analysis of poverty. Methodological and conceptual challenges to understanding poverty from a gender perspective. Santiago de Chile: Naciones Unidas, Comisión Económica para América Latina, Serie Mujer y Desarrollo, accesible en http://www.eclac.org/publicaciones/xml/6/13156/lcl1955i.pdf

Chant, Sylvia H. (2006). «Re-thinking the «feminization of poverty» in relation to aggregate gender indices», en Journal of buman development, 7 (2): 201-220, accesible en http://eprints.lse.ac.uk/2869/1/Re-thinking_the_feminisation_of_ poverty_\%28LSERO\%29.pdf

Chen, Shaohua y Ravallion, Martin (2008). «The developing world is poorer than we thought, but no less successful in the fight against poverty», en The World Bank, Policy Research Working Paper, 4703, accesible en http //www-wds.worldbank. org/external/default/WDSContentServer/IW3P/IB/2008/08/26/000158349_20 080826113239/Rendered/PDF/WPS4703.pdf

Centro de Investigaciones Sociológicas (CIS) (2008). Barómetro de diciembre de 2008, disponible en http://www.cis.es/cis/opencms/ES/index.html

Comisión de la Condición Jurídica y Social de la Mujer (2009). Nuevas cuestiones, tendencias y criterios respecto de los problemas que afectan a la situación de la mujer o la igualdad entre el hombre y la mujer. Perspectivas de género de la crisis financiera. Naciones Unidas, Seguimiento de la Cuarta Conferencia Mundial sobre la Mujer, accesible en http://www.un.org/womenwatch/daw/csw/csw53/crps/E-CN.6-2009CRP.7\%20Sp.pdf 
Comisión Europea (2009). Joint report on social protection and social inclusion 2009. Dirección general para el Empleo, Asuntos Sociales e Igualdad de Oportunidades, Luxemburgo: Comisión Europea.

Consejo de Europa (2005). Elaboration concertée des indicateurs de la cohésion sociale. Guide méthodologique. Estrasburgo, Éditions du Conseil de l'Europe.

Dalaker, Joe (2005). «Alternative poverty estimates in the United States: 2003, U.S. Census Bureau», en Current Population Reports, junio de 2005, accesible en http:// www.censusbureau.biz/prod/2005pubs/p60-227.pdf\#page $=1$

Davids, Tine y Van Driel, Francien (2005). «Changing perspectives», en DAvids, Tine y Van Driel, Francien (ed.) (2005) The gender question in globalisation. Aldershot: Ashgate.

Dennis, Ian y Guio, Anne-Catherine (2004). Monetary poverty in new member states and candidate countries. Statistics in focus, Eurostat, 12:1-11.

Elejabeitia, Carmen de (1993). «Feminización de la pobreza», en Documentación social, 105: 171-182.

Espinar, Eva y Mateo, Miguel Ángel (2007). «Violencia de género: reflexiones conceptuales, derivaciones prácticas», en Papers, Revista de Sociología, 86: 189-201.

EUROSTAT (2008). Living conditions in Europe. Data 2003-2006. Edición de 2008, Luxemburgo: Eurostat, Comunidad Europea, accesible en http://epp.eurostat. ec.europa.eu/cache/ITY_OFFPUB/KS-DZ-08-001/EN/KS-DZ-08-001-EN. PDF

GALLUP (2008). Even before crisis, affording food a challenge for many. Publicado el 25 de abril de 2008, accesible en http://www.gallup.com/poll/106807/Even-BeforeCrisis-Affording-Food-Challenge-Many.aspx

GALLUP (2009). Eating well and life satisfaction: A global view. Publicado el 16 de enero de 2009, accesible en http://www.gallup.com/poll/113827/Eating-Well-LifeSatisfaction-Global-View.aspx

Hausmann, Richard; Tyson, Laura D.; Zahidi, Saadia (2008), The Global Gender Gap Report, Ginebra: World Economic Forum, accesible en http://www.weforum. org/pdf/gendergap/report2008.pdf

INE (2008). Encuesta de Condiciones de Vida, 2007, Madrid: Instituto Nacional de Estadística, accesible en http://www.ine.es/prensa/np526.pdf

Martínez Román, María Asunción (2001). «Género, pobreza y exclusión: diferentes conceptualizaciones y políticas públicas», en TorTosA, José María (coord.) (2001), Pobreza y perspectiva de género. Barcelona: Icaria

Mateo, Miguel Ángel (2000)* «Dos perspectivas metodológicas para la inclusión de la perspectiva de género en el análisis de la pobreza», en Psicothema. 12 (2) 377-381. 
Mateo, Miguel Ángel (2001). «Desigualdad, pobreza y exclusión: Conceptos, medidas y alternativas metodológicas», en TortosA, José María (coord.) (2001), Pobreza $y$ perspectiva de género. Barcelona: Icaria.

Mateo, Miguel Ángel (2002). «¿Conoces a alguien más pobre que yo? Definiciones de la pobreza desde la perspectiva de género», en Tortosa, José María (coord.) (2002), Mujeres pobres: Indicadores de empobrecimiento en la España de hoy. Madrid: FOESSA.

Medeiros, Marcelo y Costa, Joana (2008). «Is there a feminization of poverty in Latin America?», en World Development, 36 (1): 115-127.

Naciones Unidas (2005). Millenium Development Goals Report 2005. Nueva York: Naciones Unidas, disponible en http://www.un.org/docs/summit2005/MDGBook.pdf

Naciones Unidas (2007). Informe 2007 sobre los ODM. Nueva York: Naciones Unidas, accesible en http://mdgs.un.org/unsd/mdg/Resources/Static/Products/ Progress2007/UNSD_MDG_Report_2007e.pdf

Observatorio de la Inclusión Social (2008). Informe sobre la inclusión social en España 2008. Barcelona: Caixa Catalunya, Obra Social, accesible en http://obrasocial.caixacatalunya.es/osocial/idiomes/2/fitxers/solidaritat/informe_incl08cas.pdf

Pérez Yruela, Manuel et alii (Instituto de Estudios Sociales Avanzados IESACSIC) (2009). Condiciones de vida y pobreza relativa de la población de Aragón. Zaragoza: Consejo Económico y Social de Aragón.

PNUD (2007). Informe sobre el desarrollo bumano 2007/2008. Programa de Naciones Unidas para el Desarrollo, accesible en http://hdr.undp.org/en/media/ HDR_20072008_SP_Complete.pdf

Ravallion, Martin; Chen, Shaohua, y Sangraula, Prem (2008). «Dollar a day revisited», en The World Bank, Policy Research Working Paper 4620, accesible en http://www-wds.worldbank.org/external/default/WDSContentServer/IW3P/IB/ 2008/05/19/000158349_20080519155817/Rendered/PDF/wps4620.pdf

Save the Children (2009). State of the World's Mothers, 2009. Saving the lives of Children under 5. Accesible en http://www.infoplease.com/spot/mothersindex.html

Thibos, Megan; Lavin-Loucks, Danielle, y Martin, Marcus (2007). The feminization of poverty. YWCA y The Williams Institute, accesible en http://www.dallasindicators.org/Portals/8/Reports/Reports_Internal/Feminization\%20of\%20Poverty.pdf

Townsend, Peter (1979). Poverty in the United Kingdom. Harmondsworth: Penguin Books.

Townsend, Peter (1987). «Deprivation», en Journal of Social Policy,16 (2) 125-46. 
UNIFEM (2008). El Progreso de las mujeres en el mundo 2008/2009: ¿Quién responde a las mujeres? Género y rendición de cuentas. Nueva York: Fondo de Desarrollo de las Naciones Unidas para la Mujer, accesible en http://www.unifem.org/progress/2008

U.S. Census Bureau (2009). Poverty, accesible en http://www.census.gov/hhes/ www/poverty/poverty07.html

VAldés, Ximena S. (2009). «El lugar que habita el padre en Chile contemporáneo. Estudio de las representaciones sobre la paternidad en distintos grupos sociales», en Revista Polis, 23, accesible en http://www.revistapolis.cl/polis\%20final/23/art16.htm VV.AA. (2007). Poverty: An international glossary. Second edition. Londres: Zed Books. 